\title{
Search for new cosmic-ray acceleration sites within the 4FGL Catalog sources
}

\section{Lemoine-Goumard ${ }^{a * *}$ and J. Ballet ${ }^{b}$ for the Fermi-LAT collaboration}

${ }^{a}$ Univ. Bordeaux, CNRS, CENBG, UMR 5797, F-33170 Gradignan, France

${ }^{b}$ AIM, CEA, CNRS, Université Paris-Saclay, Université de Paris, F-91191 Gif sur Yvette, France

E-mail: lemoine@cenbg.in2p3.fr

Cosmic rays are mostly composed of protons accelerated to relativistic speeds. When those protons encounter interstellar material, they produce neutral pions which in turn decay into gamma rays. This offers a compelling way to identify the acceleration sites of protons. A characteristic hadronic spectrum was detected in the gamma-ray spectra of four Supernova Remnants (SNRs), IC 443, W44, W49B and W51C, with the Fermi Large Area Telescope. This detection provided direct evidence that cosmic-ray protons are (re-)accelerated in SNRs.

In this review, we present the results from a comprehensive search for low energy spectral breaks. We use 8 years of data from the Fermi Large Area Telescope between $50 \mathrm{MeV}$ and $1 \mathrm{GeV}$. This search is based on the 4FGL Catalog from which we extracted the unidentified sources or those associated to SNRs with a significance above $3 \sigma$ at low energy in both cases. Several SNRs, binaries and one star forming region as well as a handful of unidentified sources are detected with our search. These best candidates will be presented, thus enlarging our view to potential new cosmic-ray acceleration sites.

$37^{\text {th }}$ International Cosmic Ray Conference (ICRC 2021)

July 12th - 23rd, 2021

Online - Berlin, Germany

\footnotetext{
${ }^{*}$ Presenter
} 


\section{Introduction}

More than one century after their discovery, the origin of cosmic rays (CRs) is still a crucial issue in high-energy astrophysics. According to a popular hypothesis, Galactic CRs are accelerated at SuperNova Remnant (SNR) shocks through the diffusive shock acceleration mechanism [e.g. 1]. Verification of this hypothesis is possible by studying the $\gamma$-ray emission from SNRs. Indeed, accelerated high energy protons interact with surrounding matter and produce $\pi^{0}$ mesons which usually quickly decay into two gamma rays, each having an energy of $67.5 \mathrm{MeV}$ in the rest frame of the neutral pion. This leads to a $\gamma$-ray number spectrum, $F(E)$, symmetric about $67.5 \mathrm{MeV}$ in a $\log$ - $\log$ representation. In turn, the usual $E^{2} F(E)$ representation presents a characteristic break at $E \sim 200-300 \mathrm{MeV}$. This characteristic spectral feature (referred to as the "pion-decay bump") uniquely identifies $\pi^{0}$-decay gamma rays and thereby high-energy protons, allowing to identify cosmic-ray accelerators. Such detection was achieved for five SNRs interacting with molecular clouds (MCs) detected at $\gamma$-ray energies by Fermi-LAT: IC 443 and W44 [2, 3], W49B [4], W51C [5] and HB 21 [6], although in this last source both the leptonic and hadronic processes are able to reproduce the $\gamma$-ray emission. Finally, the young SNR Cassiopeia A was also analyzed at low energy and [7] derived an energy break at $1.7 \mathrm{GeV}$ which is better reproduced by a hadronic scenario.

Here, we tried to find new cosmic-ray acceleration sites in our Galaxy by looking for characteristic pion-decay bumps in the LAT data. For this search, we selected 311 sources detected in the 4FGL Catalog [8] within $5^{\circ}$ from the Galactic plane with a significance above $3 \sigma$ between $300 \mathrm{MeV}$ and $1 \mathrm{GeV}$ in the Catalog, removing identified extragalactic sources (except UNK-class sources which refer to sources associated solely via the Likelihood-Ratio method to counterparts of unknown type in large catalogs) and pulsars.

\section{Fermi-LAT data analysis}

For this analysis, we have used the exact same dataset prepared in the framework of the 4FGL Catalog, i.e 8 years (from 2008 August 4 to 2016 August 2) of Fermi-LAT Pass 8 data between $50 \mathrm{MeV}$ and $1 \mathrm{GeV}$. We used the Source class event selection, with the current version of the Instrument Response Functions (IRFs) P8R3_SOURCE_V3. The Galactic diffuse emission was modeled by the standard file gll_iem_v07.fits and the residual background and extragalactic radiation were described by a single isotropic component with the spectral shape in the tabulated model iso_P8R3_SOURCE_V3_v1.txt.

Due to the poor angular resolution below $100 \mathrm{MeV}$, we decided to use only PSF3 events (the events with the best angular resolution) below this energy. We add PSF2 events between $100 \mathrm{MeV}$ and $1 \mathrm{GeV}$. This high-energy bound was selected since middle-aged SNRs commonly exhibit a highenergy spectral break at a few GeV which would then bias our low-energy analysis [9]. The data reduction and exposure calculations were performed using the LAT fermitools version 1.2.23 and fermipy [10] version 0.19.0. We used only binned likelihood analysis because unbinned mode is much more CPU intensive and does not support energy dispersion.

We accounted for the effect of energy dispersion (reconstructed event energy not equal to the true energy of the incoming $\gamma$-ray) by using edisp_bins $=-3$ which means that the energy dispersion 
correction operates on the spectra with three extra bins below and above the threshold of the analysis ${ }^{1}$.

\section{Detection of low-energy breaks}

We performed an independent analysis of the 311 candidates, following a procedure developed for the Fermi High-Latitude Extended Sources Catalog [11], which already used the functions provided by fermipy.

For each source of interest, we define a $20^{\circ} \times 20^{\circ}$ region and include in our baseline model all 4FGL sources located in a $40^{\circ} \times 40^{\circ}$ region centered on the source, using the same spectral and spatial parameterization used in the 4FGL Catalog. We first optimize each region by re-fitting the normalizations of all sources in the order of decreasing total predicted counts in the model (Npred) down to Npred $=1$. We conclude this optimization step by iteratively re-fitting the spectral parameters of all sources down to TS $=25$. We then identify new point sources with TS $>16$ by generating a TS map for a point source that has a PL spectrum with an index $\Gamma=2$. We fit the spectrum and position of each source added.

Once the ROI is well characterized, we first test the TS value of the source of interest in our energy range (50 MeV to $1 \mathrm{GeV}$ ). If it is below 25 , we stop the analysis for this source since it is not significantly detected in our pipeline. If the TS of the source of interest is above 25 , we test the curvature of its spectrum by estimating the improvement of the log-normal representation with respect to the power-law model: $\mathrm{TS}_{\mathrm{LP}}=2\left(\ln \mathcal{L}_{\mathrm{LP}}-\ln \mathcal{L}_{\mathrm{PL}}\right)$. If $\mathrm{TS}_{\mathrm{LP}}$ is below 9 , we consider that no significant curvature is detected by our pipeline and we stop the analysis of this source. If the value is above 9, we then test a smoothly broken power law as was done previously for IC 443 and W44 [3]. The improvement with respect to the power law fit is determined by $\mathrm{TS}_{\mathrm{SBPL}}=2\left(\ln \mathcal{L}_{\mathrm{SBPL}}-\ln \mathcal{L}_{\mathrm{PL}}\right)$. Since this test requires the addition of two degrees of freedom to the fit (the break energy $E_{\mathrm{br}}$ and a second spectral index $\Gamma_{2}$ ) and diffusive shock acceleration predicts $\Gamma_{2} \sim 2$, we also test the improvement of the smooth broken power law with the second index fixed at 2 with respect to the power law fit $\mathrm{TS}_{\mathrm{SBPL} 2}=2\left(\ln \mathcal{L}_{\mathrm{SBPL} 2}-\ln \mathcal{L}_{\mathrm{PL}}\right)$. We require $\mathrm{TS}_{\mathrm{SBPL}}>12$ or $\mathrm{TS}_{\mathrm{SBPL} 2}>9$ (implying a $3 \sigma$ improvement for 2 and 1 additional degrees of freedom respectively) to keep the source in the list of sources showing a significant energy break at low energy. This procedure allowed the detection of 77 sources presenting a significant energy break in their low-energy spectrum.

For sources located in the Galactic plane, the dominant systematic error at energies below 1 $\mathrm{GeV}$ arises from the uncertainty in the model of the Galactic diffuse emission. To test for this effect, we repeated our analysis for the 77 sources using the old diffuse rescaled for Pass 8 Source gll_iem_v06.fits. For each source, we tested if $\mathrm{TS}_{\mathrm{SBPL}}>12$ or $\mathrm{TS}_{\mathrm{SBPL} 2}>9$. If it was not the case, then this source was discarded from the final list of sources presenting a significant energy break.

An additional important source of systematic errors that needs to be considered is the effect of the inaccuracies in the effective area. To quantify their effect, we refit each candidate with each of the two bracketing IRFs ${ }^{2}$ and again tested if $\mathrm{TS}_{\mathrm{SBPL}}>12$ or $\mathrm{TS}_{\mathrm{SBPL} 2}>9$.

Overall, 56 sources among the 77 sources detected with the standard interstellar emission model and IRFs are confirmed with our systematic studies. We computed a spectral energy distribution

${ }^{1}$ https://fermi.gsfc.nasa.gov/ssc/data/analysis/documentation/Pass8_edisp_usage.html

${ }^{2}$ https://fermi.gsfc.nasa.gov/ssc/data/analysis/scitools/Aeff_Systematics.html 
(SED) for each of them by fitting the flux of the source independently in 10 energy bins spaced uniformly in log space from $50 \mathrm{MeV}$ to $1 \mathrm{GeV}$. The systematic studies with the old diffuse and bracketing IRFs were also performed on all SED points and the two uncertainties were added in quadrature.
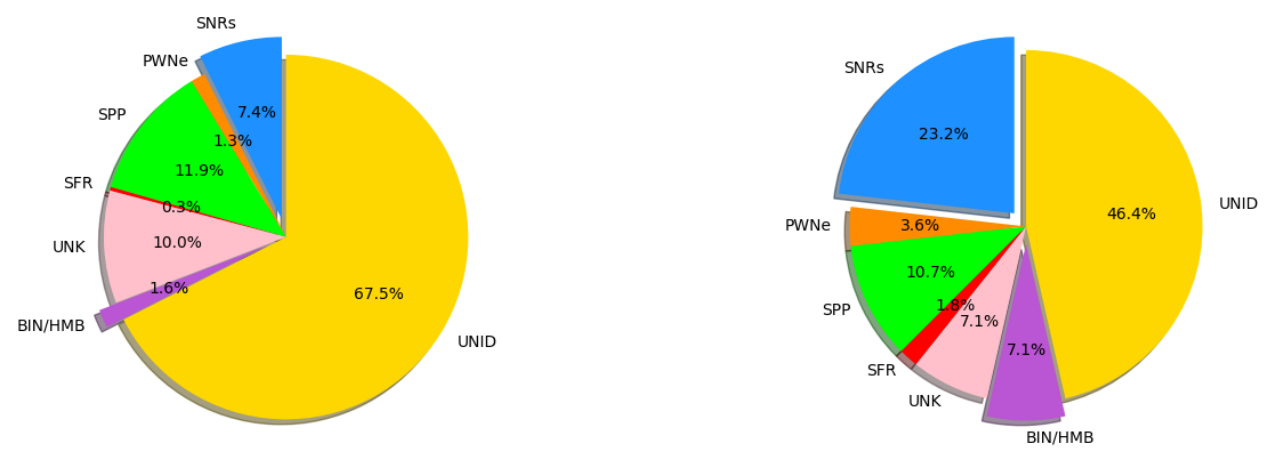

Figure 1: Pie charts showing the classes of sources analyzed (left) and those for which a significant break is detected (right). The class names are those used in the 4FGL Catalog: SNR stands for Supernova remnant, PWN for pulsar wind nebula, SFR for star-forming region, BIN for binary, HMB for high-mass binary. The designation SPP indicates potential association with SNR or PWN. The UNK class includes low-latitude sources of unknown type associated solely via the Likelihood-Ratio (LR) method.

\section{Population study}

The association summary is illustrated by the pie charts in Figure 1. Out of 311 candidates, 210 are unidentified, representing $67.5 \%$ of the sources analyzed. It is striking to see that only 26 UNIDs show a spectral break confirmed with our systematic studies (which represents $46.4 \%$ of these sources). On the other hand, the fraction of sources associated with supernova remnants (SNRs) increases from $7.4 \%$ (23 out of 311 sources) to $23.2 \%$ (13 out of 56 sources). This makes SNRs the dominant class of sources with a significant low-energy spectral break. Similarly, the fraction of sources associated with binaries increases from 1.6\% (5 out of 311) to 7.1\% (4 out of 56). However, there might be a selection bias here since identified sources tend to have higher TS value than unidentified sources and a spectral break is therefore more likely to be detected for the latter.

Figure 2 illustrates the distribution over the sky of the 56 4FGL $\gamma$-ray sources showing a significant energy break. The distribution of the longitude of these sources does not show a clear trend. The lack of sources at latitude smaller than $-2^{\circ}$ appears clearly. One can also note a large number of unidentified sources at longitude comprised between $-50^{\circ}$ and $50^{\circ}$. These sources are part of the large fraction of 4FGL unassociated sources located less than $10^{\circ}$ away from the Galactic plane with a wide latitude extension hard to reconcile with those of known classes of Galactic $\gamma$-ray sources [12]. 


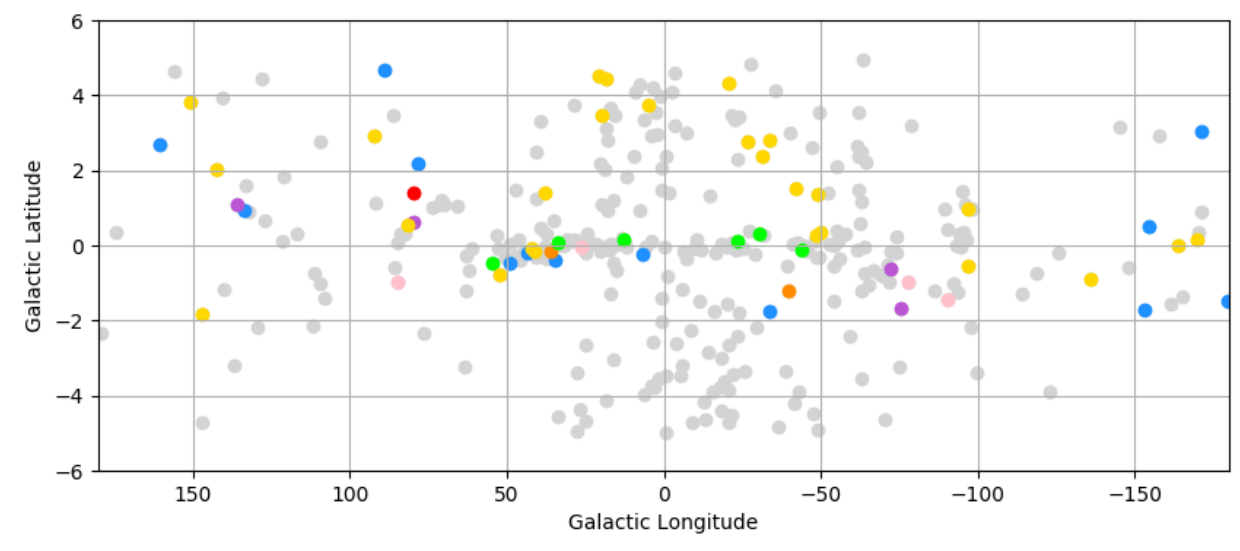

Figure 2: Distribution of sources in Galactic coordinates. Light gray markers indicate the 311 sources analyzed in this paper. Coloured markers indicate the positions of the 56 sources for which a significant break is detected: yellow for UNIDs, blue for SNRs, orange for PWNe, green for SPPs, red for SFR, pink for UNKs and purple for BIN/HMB. The boundary of the latitude selection is $5^{\circ}$. The vertical scale $($ in $\% \mathrm{~cm})$ is about 10 times larger than the horizontal one.

\section{Supernova remnants and molecular clouds}

The dominant class of identified sources reported in our analysis (as illustrated in Figure 1) is SNRs with ten firm SNR identifications and three SNR associations. The first sources for which the characteristic "pion-decay bump" feature was detected were the middle-aged remnants IC 443 and W44. With no surprise, these two SNRs are among the sources detected in our analysis that show a significant break at low energy. Figure 3 presents the residual TS map of the region of IC 443 (4FGL J0617.2+2234e) as well as its SED, showing the overall agreement with the 4FGL SED points superimposed. This Figure also illustrates the advantages of using a restricted energy range and different spectral shape than the 4FGL to better reproduce the significant energy break at low energy since we are not dominated here by photons at high energies. More generally, all the SNRs for which a spectral break has been published are detected in our pipeline except Cassiopeia A (4FGL J2323.4+5849). However, the break energy reported by [7] is at $1.72_{-0.89}^{+1.35} \mathrm{GeV}$ which seems consistent with our non-detection in the $50 \mathrm{MeV}-1 \mathrm{GeV}$ energy interval.

\section{Other cosmic-ray accelerators}

No observational evidence for the acceleration of PeV CRs at SNRs has ever been found, and theoretical predictions struggle to reach PeV energies [13]. SNRs might indeed act as proton accelerators, but would be PeVatrons only during a very short early phase lasting several tens of years after the supernova explosion. Other types of Galactic accelerators might contribute significantly to the CR spectrum. The shocks generated by the stellar winds of massive stars or star-forming regions are among these cosmic-ray accelerators. In this context, the detection in $\gamma$ rays of the Cygnus region by the LAT [14] opened new perspectives by revealing the presence of a cocoon of freshly-accelerated CRs over a scale of $\sim 50 \mathrm{pc}$. Our analysis reveals a spectral break for the 

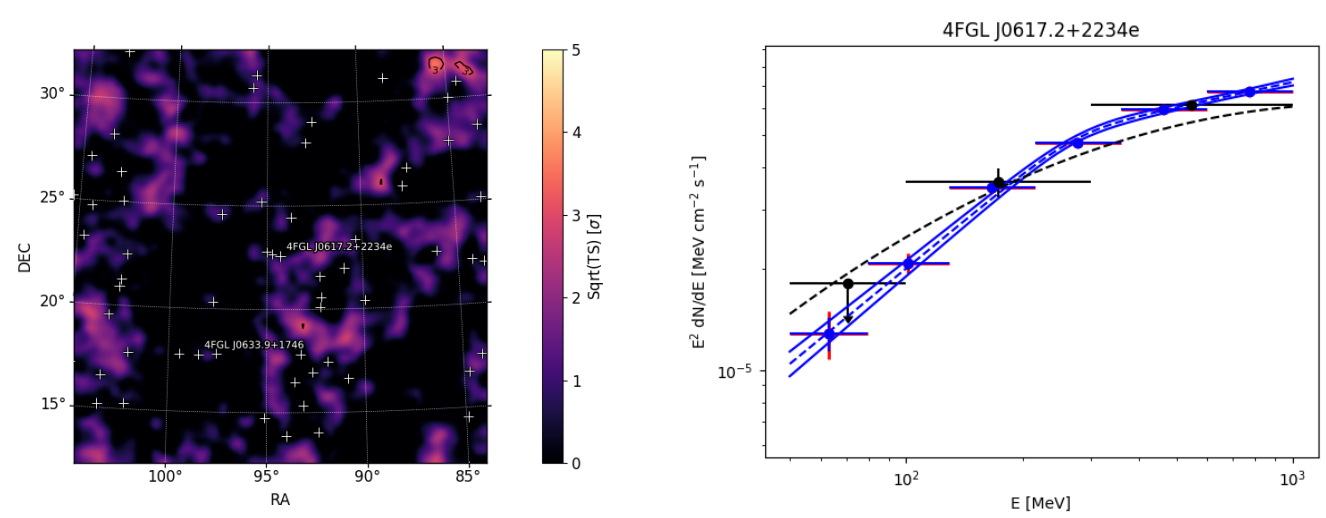

Figure 3: LAT residual TS map in equatorial coordinates and significance units (left) and spectral energy distribution (right) of IC 443 between $50 \mathrm{MeV}$ and $1 \mathrm{GeV}$. In the residual TS map, all white crosses indicate the sources added in the model of the region. For the SED, the blue points and butterflies are obtained in this analysis while the black points are from the 4FGL Catalog. The red lines take into account both the statistical and systematic errors added in quadrature. A 95\% C.L. upper limit is computed when the TS value is below 1.

star-forming region analyzed, 4FGL J2028.6+4110e, which is associated with the cocoon. Figure 4 (left) presents the spectrum derived for this source, which is similar to those observed for the population of interacting SNRs with a hard index detected up to a break energy at $\sim 400 \mathrm{MeV}$ followed by a spectral index $\Gamma_{2} \sim 2.2$. However, this is the only identified star forming region included in the sample of 311 sources analyzed, which prevents us from drawing any conclusion on their impact for the Galactic CR spectrum. Gamma-ray binaries, microquasars and colliding wind binaries could also contribute to the sea of Galactic cosmic rays. This is well illustrated by Figure 4 (right) which presents the spectrum derived for 4FGL J1045.1-5940 associated with the colliding wind binary $\eta$ Carinae. Again, the SED derived is very similar to those obtained for interacting SNRs.
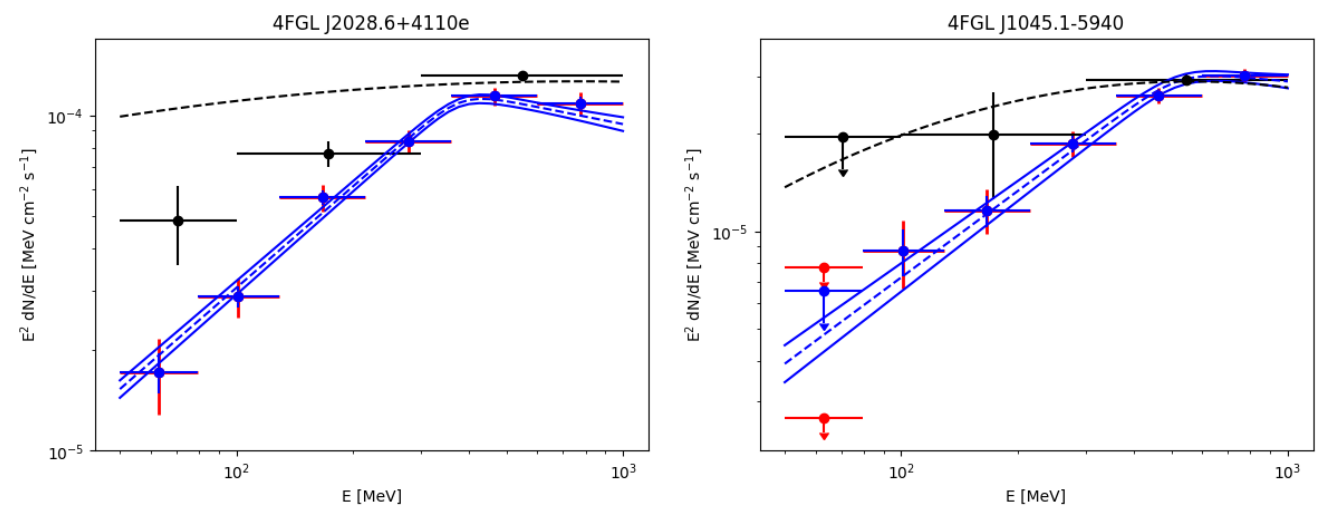

Figure 4: LAT spectral energy distributions of 4FGL J2028.6+4110e (Cygnus X cocoon, left) and 4FGL J1045.1-5940 ( $\eta$ Car, right) with the same conventions used in Figure 3. 


\section{Conclusion}

Using 8 years of Pass 8 Fermi-LAT data between $50 \mathrm{MeV}$ and $1 \mathrm{GeV}$, we have analyzed 311 4FGL sources located within $5^{\circ}$ from the Galactic plane and detected 56 sources with significant spectral breaks which are confirmed by our thorough systematic studies. We computed an SED for each of these 56 sources. With 13 SNRs identified within this sample of 56 sources, SNRs are the dominant class of sources showing significant breaks at low energy. But other $\gamma$-ray sources could contribute to the sea of Galactic cosmic rays, such as star forming regions or $\gamma$ ray binaries. Unassociated and not well classified sources represent $29.7 \%$ of the 4FGL Catalog sources: revealing the mystery of the nature of these unidentified $\gamma$-ray sources might shed new light on the problem of the origin of Galactic CRs. In our analysis, $46.4 \%$ of the sources showing breaks are unidentified and their low-energy spectra could therefore prove very useful to constrain their nature. Obviously, to confirm the hadronic origin of the $\gamma$-ray emission detected in the 56 candidates reported by our analysis, a complete modeling of each source would be needed. Among the 56 sources showing low-energy spectral breaks, those that show hard spectra above $10 \mathrm{GeV}$ are excellent targets for current and future Cherenkov telescopes such as CTA which would then provide constraints on the maximum energy reached by the accelerated protons.

\section{Acknowledgements}

The Fermi-LAT Collaboration acknowledges support for LAT development, operation and data analysis from NASA and DOE (United States), CEA/Irfu and IN2P3/CNRS (France), ASI and INFN (Italy), MEXT, KEK, and JAXA (Japan), and the K.A. Wallenberg Foundation, the Swedish Research Council and the National Space Board (Sweden). Science analysis support in the operations phase from INAF (Italy) and CNES (France) is also gratefully acknowledged. This work performed in part under DOE Contract DEAC02-76SF00515.

\section{References}

[1] Drury, L. O. 1983, Reports on Progress in Physics, 46, 973

[2] Giuliani, A. et al. 2011, ApJL, 742, L30, 1111.4868

[3] Ackermann, M. et al. 2013, Science, 339, 807, 1302.3307

[4] H. E. S. S. Collaboration et al. 2018, A\&A, 612, A5, 1609.00600

[5] Jogler, T., \& Funk, S. 2016, ApJ, 816, 100

[6] Ambrogi, L., Zanin, R., Casanova, S. et al. 2019, A\&A, 623, A86, 1902.06064

[7] Yuan, Y., Funk, S., Johannesson, G. et al., 2013, ApJ, 779, 117, 1310.8287

[8] Abdollahi, S. et al. 2020, ApJS, 247, 33, 1902.10045

[9] Uchiyama, Y., Blandford, R. D., Funk, S. et al. 2010, ApJL, 723, L122, 1008.1840

[10] Wood, M., Caputo, R., Charles, E. et al., 2017 in International Cosmic Ray Conference, Vol. 301, 35th, $824,1707.09551$ 
[11] Ackermann, M. et al. 2018, ApJS, 237, 32, 1804.08035

[12] Lott B., Ballet J., Bruel P. and Burnett T., this conference

[13] Schure K. M., Bell A. R., 2013, MNRAS, 435, 1174

[14] Ackermann, M. et al. 2011, Science, 334, 1103 